\title{
A VERY SIMPLE CHARACTERIZATION OF GROMOV HYPERBOLICITY FOR A SPECIAL KIND OF DENJOY DOMAINS
}

\author{
Ana Portilla ${ }^{\dagger, \ddagger}$, José M. RodríGuez ${ }^{\ddagger}$, And Eva Tourís ${ }^{\dagger, \ddagger, *}$ \\ ABSTRACT. In this paper we provide characterizations for the Gromov \\ hyperbolicity of some particular Denjoy domains and besides some suffi- \\ cient conditions to guarantee or discard the hyperbolicity of some others. \\ The conditions obtained involve just the lengths of some special simple \\ closed geodesics in the domain. These results, on the one hand, show the \\ extraordinary complexity of determining the hyperbolicity of a domain \\ and, on the other hand, allow us to construct easily a large variety of \\ both hyperbolic and non-hyperbolic domains.
}

\section{Introduction}

In the 1980s Mikhail Gromov introduced a notion of abstract hyperbolic spaces, which have thereafter been studied and developed by many authors. Initially, the research was mainly centered on hyperbolic group theory, but lately researchers have shown an increasing interest in more direct studies of spaces endowed with metrics used in geometric function theory.

To understand the connections between graphs and potential theory on Riemannian manifolds (see e.g. [4], [12], [14], [27], [28], [29], [30], [40], [41], [46], [47]), Gromov hyperbolic spaces are a useful tool. Besides, the concept of Gromov hyperbolicity grasps the essence of negatively curved spaces, and has been successfully used in the theory of groups (see e.g. [13], [16], [18], [19] and the references therein).

Received January 29, 2010; Revised August 16, 2010.

2010 Mathematics Subject Classification. Primary 30F45; Secondary 53C23, 30C99.

Key words and phrases. Denjoy domain, Gromov hyperbolicity, Poincaré metric, train.

$\dagger$ Supported in part by a grant from Ministerio de Ciencia e Innovación (MTM 200912740-C03-01), Spain.

$\ddagger$ Supported in part by two grants from Ministerio de Ciencia e Innovación (MTM 200907800 and MTM 2008-02829-E), Spain.

* Supported in part by a grant from U.C.III M./C.A.M. (CCG08-UC3M/ESP-4516), Spain.

(C)2011 The Korean Mathematical Society 
One of the primary questions is naturally whether a metric space $(X, d)$ is hyperbolic in the sense of Gromov or not. The most classical examples, mentioned in every textbook on this topic, are metric trees, the classical Poincaré hyperbolic metric developed in the unit disk and, more generally, simply connected complete Riemannian manifolds with sectional curvature $K \leq-k^{2}<0$.

However, it is not easy to determine whether a given space is Gromov hyperbolic or not. In recent years several investigators have been interested in showing that metrics used in geometric function theory are Gromov hyperbolic. For instance, the Klein-Hilbert metric (see [10, 31]) is Gromov hyperbolic (under particular conditions on the domain of definition); the Gehring-Osgood $j$-metric (see [21]) is Gromov hyperbolic; and the Vuorinen $j$-metric (see [21]) is not Gromov hyperbolic except in the punctured space. Also, in [32] the hyperbolicity of the conformal modulus metric $\mu$ and the related so-called Ferrand metric $\lambda^{*}$, is studied.

The quasihyperbolic metric has also recently been a topic of interest regarding the question of Gromov hyperbolicity. In [11], Bonk, Heinonen and Koskela found necessary and sufficient conditions for when a planar domain $D$ endowed with the quasihyperbolic metric is Gromov hyperbolic. This was extended by Balogh and Buckley, [6]: they found two different necessary and sufficient conditions which work in Euclidean spaces of all dimensions and also in metric spaces under some conditions.

Since the Poincaré metric is also the metric giving rise to what is commonly known as the hyperbolic metric when speaking about open domains in the complex plane or in Riemann surfaces, it could be expected that there is a connection between the notions of hyperbolicity. For simply connected subdomains $\Omega$ of the complex plane, it follows directly from the Riemann mapping theorem that $\Omega$ is, in fact, Gromov hyperbolic. However, as soon as simple connectedness is omitted, there is no immediate answer to whether the space $\Omega$ is hyperbolic or not. The question has lately been studied in [3], [22]-[26], [34]-[45] and [47].

In the current paper our main aim is to study the Gromov hyperbolicity of Denjoy domains, that is to say, plane domains $\Omega$ with $\partial \Omega \subset \mathbb{R}$, with the Poincaré metric. This kind of surfaces are becoming more and more important in Geometric Theory of Functions, since, on the one hand, they are a very general type of Riemann surfaces, and, on the other hand, they are more manageable due to its symmetry. For instance, Garnett and Jones have proved in [15] the Corona Theorem for Denjoy domains, and in [2], [42] the authors have got characterizations of Denjoy domains which satisfy a linear isoperimetric inequality.

The Gromov hyperbolicity of Denjoy domains with the Poincaré and quasihyperbolic metrics has been studied previously in [22], [23] and [24] in terms of the Euclidean size of the boundary of the Denjoy domain. The same topic, just for the Poincaré metric, has been dealt with in [3] and [38], but from a geometric point of view. 
In this paper we provide new and easily applicable geometric criteria (involving the lengths of some kind of closed geodesics) in order to guarantee or discard Gromov hyperbolicity of some Denjoy domains.

First of all we find a very simple characterization of Gromov hyperbolicity for a special kind of Denjoy domains (see Theorem 3.1). If we enlarge slightly this class, the situation becomes extraordinarily complicated (see Theorem 3.9 for sufficient conditions on non-hyperbolicity and Theorem 3.10 for a sufficient condition on hyperbolicity); these results allow to construct easily a large variety of both hyperbolic and non-hyperbolic domains. Again, by imposing certain regularity condition on these domains, we get a characterization (see Theorem $4.3)$.

Theorem 4.3 reduces the study of such a complicated topic as hyperbolicity to something as simple as checking the boundedness or the limit of some sequences. The complexity of the statement of Theorem 4.3 shows the difficulty of the problem, but it turns out to be really easy to apply in practice.

Notations. We denote by $X$ a geodesic metric space. By $d_{X}$ and $L_{X}$ we shall denote, respectively, the distance and the length in the metric of $X$. From now on, when there is no possible confusion, we will not write the subindex $X$. We denote by $\Re z$ and $\Im z$ the real and imaginary part of $z$, respectively. We denote by $\Omega$ a Denjoy domain with its Poincaré metric. Finally, we denote by $c$ and $c_{i}$, positive constants which can assume different values in different theorems.

\section{Background}

We denote by $\mathbb{H}$ the upper half plane, $\{z \in \mathbb{C}: \Im z>0\}$ and by $\mathbb{D}$ the unit disk $\{z \in \mathbb{C}:|z|<1\}$. Recall that a domain $\Omega \subset \mathbb{C}$ is said to be of nonexceptional if it has at least two finite boundary points. The universal cover of such domain is the unit disk $\mathbb{D}$. In $\Omega$ we can define the Poincaré metric, i.e., the metric obtained by projecting the metric $d s=2|d z| /\left(1-|z|^{2}\right)$ of the unit disk by any universal covering map $\pi: \mathbb{D} \longrightarrow \Omega$. Equivalently, we can project the metric $d s=|d z| / \Im z$ of the upper half plane $\mathbb{H}:=\{z \in \mathbb{C}: \Im z>0\}$. Therefore, any simply connected subset of $\Omega$ is isometric to a subset of $\mathbb{D}$. With this metric, $\Omega$ is a geodesically complete Riemannian manifold with constant curvature -1 ; in particular, $\Omega$ is a geodesic metric space (see Definition 2.1 below). The Poincaré metric is natural and useful in complex analysis; for instance, any holomorphic function between two domains is Lipschitz with constant 1, when we consider the respective Poincaré metrics.

A Denjoy domain $\Omega \subset \mathbb{C}$ is a domain whose boundary is contained in the real axis. Since $\Omega \cap \mathbb{R}$ is an open set contained in $\mathbb{R}$, it is the union of pairwise disjoint open intervals; as each interval contains a rational number, this union is countable. Hence, we can write $\Omega \cap \mathbb{R}=\cup_{n \in \Lambda}\left(a_{n}, b_{n}\right)$, where $\Lambda$ is a countable index set, $\left\{\left(a_{n}, b_{n}\right)\right\}_{n \in \Lambda}$ are pairwise disjoint, and it is possible to have $a_{n_{1}}=$ $-\infty$ for some $n_{1} \in \Lambda$ and/or $b_{n_{2}}=\infty$ for some $n_{2} \in \Lambda$. 
In order to study Gromov hyperbolicity, we consider the case where $\Lambda$ is countably infinite, since if $\Lambda$ is finite, then $\Omega$ is Gromov hyperbolic by [22, Proposition 3.6] or [42, Proposition 3.2].

As we mentioned in the introduction of this paper, Denjoy domains are becoming more and more interesting in Geometric Function Theory (see e.g. [1], [2], [15], [17], [42]). In particular, they contain the class of flute surfaces (see, e.g. [7], [8]); these ones are important since they are the simplest examples of infinite ends, and besides, in a flute surface it is possible to give a fairly precise description of the ending geometry (see, e.g. [20]).

We collect some basic facts on Gromov hyperbolicity (see e.g. [13] and/or [16] for deeper background).

Definition 2.1. If $\gamma:[a, b] \longrightarrow X$ is a continuous curve in a metric space $(X, d)$, the length of $\gamma$ is

$$
L(\gamma):=\sup \left\{\sum_{i=1}^{n} d\left(\gamma\left(t_{i-1}\right), \gamma\left(t_{i}\right)\right): a=t_{0}<t_{1}<\cdots<t_{n}=b\right\} .
$$

We say that $\gamma$ is a geodesic if it is an isometry, i.e., $L\left(\left.\gamma\right|_{[t, s]}\right)=d(\gamma(t), \gamma(s))=$ $|t-s|$ for every $s, t \in[a, b]$. We say that $X$ is a geodesic metric space if for every $x, y \in X$ there exists a geodesic joining $x$ and $y$; we denote by $x y$ any of such geodesics (since we do not require uniqueness of geodesics, this notation is ambiguous, but convenient as well).

Definition 2.2. If $X$ is a geodesic metric space and $J$ is a polygon whose sides are $J_{1}, J_{2}, \ldots, J_{n}$, we say that $J$ is $\delta$-thin if for every $x \in J_{i}$ we have that $d\left(x, \cup_{j \neq i} J_{j}\right) \leq \delta$. We say that a polygon is geodesic if all of its sides are geodesics. The space $X$ is $\delta$-thin (or $\delta$-hyperbolic) if every geodesic triangle in $X$ is $\delta$-thin.

Remark 2.3. If $X$ is $\delta$-thin, it is easy to check that every geodesic polygon with $n$ sides is $(n-2) \delta$-thin. In particular, since $\mathbb{H}$ is $\delta_{0}$-thin, with $\delta_{0}:=\log (1+\sqrt{2})$ (see, e.g. [5, p. 130]), every geodesic hexagon in $\mathbb{H}$ is $4 \delta_{0}$-thin; more generally, every simply connected geodesic hexagon in a Riemann surface endowed with its Poincaré metric is $4 \delta_{0}$-thin, since it is isometric to a geodesic hexagon in $\mathbb{H}$.

\section{Example 2.4.}

(1) Every bounded metric space $X$ is (diam $X)$-hyperbolic (see e.g. [16, p. 29]).

(2) Every complete simply connected Riemannian manifold with sectional curvature which is bounded from above by $-k$, with $k>0$, is hyperbolic (see e.g. [16, p. 52]).

(3) Every tree with edges of arbitrary length is 0-hyperbolic (see e.g. [16, p. 29]).

In order to prove our results, we will need the following theorems. 
Theorem 2.5 ([3, Theorem 5.1]). Let $\Omega \subset \mathbb{C}$ be a Denjoy domain with $\Omega \cap \mathbb{R}=$ $\cup_{n=0}^{\infty}\left(a_{n}, b_{n}\right)$, and for each $n \geq 1$, consider a fixed geodesic $\gamma_{n}$ joining $\left(a_{0}, b_{0}\right)$ with $\left(a_{n}, b_{n}\right)$. Then, $\Omega$ is $\delta$-hyperbolic if and only if there exists a constant $c$ such that $d_{\Omega}(z, \mathbb{R}) \leq c$ for every $z \in \cup_{n} \gamma_{n}$.

Furthermore, if $\Omega$ is $\delta$-hyperbolic, then $c$ is a constant which only depends on $\delta$. If $d_{\Omega}(z, \mathbb{R}) \leq c$ for every $z \in \cup_{n} \gamma_{n}$, then $\Omega$ is $\delta$-hyperbolic, with $\delta$ a constant which only depends on $c$.

Remark 2.6. The hypothesis $\Omega \cap \mathbb{R}=\cup_{n=0}^{\infty}\left(a_{n}, b_{n}\right)$ is not restrictive at all: the case $\Omega \cap \mathbb{R}=\cup_{n=0}^{N}\left(a_{n}, b_{n}\right)$ is always hyperbolic, since $\Omega$ is of finite type (see e.g. [22, Proposition 3.6] or [43, Proposition 3.2]).

Definition 2.7. A train is a Denjoy domain $\Omega \subset \mathbb{C}$ with $\Omega \cap \mathbb{R}=\cup_{n=0}^{\infty}\left(a_{n}, b_{n}\right)$, such that $-\infty \leq a_{0}$ and $b_{n} \leq a_{n+1}$ for every $n$. A flute surface is a train with $b_{n}=a_{n+1}$ for every $n$.

We say that a curve in a train $\Omega$ is a fundamental geodesic if it is a simple closed geodesic which just intersects $\mathbb{R}$ in $\left(a_{0}, b_{0}\right)$ and $\left(a_{n}, b_{n}\right)$ for some $n>0$; we denote by $\gamma_{n}^{*}$ the fundamental geodesic corresponding to $n$ and $2 l_{n}:=L_{\Omega}\left(\gamma_{n}\right)$. A curve in a train $\Omega$ is a second fundamental geodesic if it is a simple closed geodesic which just intersects $\mathbb{R}$ in $\left(a_{n}, b_{n}\right)$ and $\left(a_{n+1}, b_{n+1}\right)$ for some $n \geq 0$; we denote by $\sigma_{n}$ the second fundamental geodesic corresponding to $n$ and $2 r_{n}:=L_{\Omega}\left(\sigma_{n}\right)$. If $b_{n}=a_{n+1}$, we define $\sigma_{n}$ as the puncture at this point and $r_{n}=0$.

A fundamental $Y$-piece in a train $\Omega$ is the generalized $Y$-piece in $\Omega$ bounded by $\gamma_{n}, \gamma_{n+1}, \sigma_{n}$ for some $n>0$; we denote by $Y_{n}$ the fundamental $Y$-piece corresponding to $n$. A fundamental hexagon in a train $\Omega$ is the intersection $H_{n}:=Y_{n}^{+}=Y_{n} \cap\{z \in \mathbb{C}: \Im z \geq 0\}$ for some $n>0$. We denote by $\alpha_{n}$ the length of the opposite side to $\sigma_{n}^{+}$in $H_{n}$.

We will need the following results.

Theorem 2.8 ([3, Theorem 5.17]). Let us consider a train $\Omega$ and a subsequence $\left\{n_{k}\right\}_{k}$ of natural numbers verifying $\lim _{k \rightarrow \infty} l_{n_{k}+1}=\lim _{k \rightarrow \infty} r_{n_{k}}=$ $\lim _{k \rightarrow \infty} r_{n_{k}+1}=\infty$ and $l_{n_{k}}, l_{n_{k}+2} \leq l_{n_{k}+1}+c$ for every $k$. Then $\Omega$ is not hyperbolic.

Definition 2.9. Given a train $\Omega$ and a point $z \in \Omega$, we define the height of $z$ as $h(z):=d_{\Omega}\left(z,\left(a_{0}, b_{0}\right)\right)$.

Lemma 2.10 ([3, Lemma 5.7]). Let us consider a train $\Omega$. We have $d_{\Omega}(z, w) \geq$ $|h(z)-h(w)|$ for every $z, w \in \Omega$.

Lemma 2.11 ([38, Proposition 3.9 and Remark $]$ ). Let us consider the fundamental hexagon $H_{n}$ and the point $z$ in $\gamma_{n+1}^{*} \cap H_{n}$ with $h(z)=h, l_{n+1} \geq h \geq l_{n}$ and $l_{n+1} \geq l_{0}$ for some constant $l_{0}>0$. Then

$$
d_{\Omega}\left(z,\left(a_{n}, b_{n}\right)\right) \asymp e^{-h+l_{n}}+e^{-\left(l_{n+1}-h-r_{n}\right)_{+}}+\left(r_{n}+h-l_{n+1}\right)_{+} \cdot
$$

Furthermore, the constants in the inequalities only depend on $l_{0}$. 
Lemma 2.12 ([3, Lemma 5.8]). Let us consider a train $\Omega$. If $l_{0} \leq l_{n+2} \leq l_{n+1}$ and $r_{n+1} \leq c_{1}$ for some fixed $n$, then $d_{\Omega}(z, \mathbb{R}) \leq d_{\Omega}\left(z,\left(a_{n+2}, b_{n+2}\right)\right) \leq c_{2}$ for every $z \in \gamma_{n+1}^{*}$ with $h(z) \in\left[l_{n+2}, l_{n+1}\right]$, where $c_{2}$ only depends on $c_{1}$ and $l_{0}$.

\section{Main results}

We start this section with a characterization of hyperbolicity for some Denjoy domains in terms of the lengths of fundamental geodesics.

Theorem 3.1. Let $\Omega$ be a Denjoy domain. If there exist $\left\{n_{k}\right\}_{k}$ such that $1 \leq n_{k+1}-n_{k} \leq 2$, and $\sup _{k} l_{n_{k}}<\infty$, then $\Omega$ is hyperbolic if and only if there exists $C$ such that

$$
\min \left\{l_{n_{k}+1}, r_{n_{k}}, r_{n_{k}+1}\right\} \leq C \quad \text { for every } k \text { with } n_{k+1}-n_{k}=2 .
$$

Proof. By hypothesis, the lengths $l_{n_{k}}$ of the fundamental geodesics $\gamma_{n_{k}}^{*}$ satisfy $l_{n_{k}} \leq c_{0}$ for some constant $c_{0}$.

Assume first that (3.1) holds.

Let us choose an arbitrary fundamental geodesic $\gamma_{m}^{*}$. On the one hand, if $m \in\left\{n_{k}\right\}_{k}$, then $d_{\Omega}(z, \mathbb{R}) \leq l_{m} \leq c_{0}$ for every $z \in \gamma_{m}^{*}$. On the other hand, if $m \notin\left\{n_{k}\right\}_{k}$ there exists some $k$ such that $m-1=n_{k}$ and $n_{k+1}-n_{k}=2$. Notice that if $l_{m} \leq C$, then $d_{\Omega}(z, \mathbb{R}) \leq l_{m} \leq C$ for every $z \in \gamma_{m}^{*}$. However, if $l_{m}>C$, then, by hypothesis, $\min \left\{r_{m-1}, r_{m}\right\} \leq C$. Without loss of generality we can assume that $r_{m} \leq C$. In particular, the fundamental hexagon $H_{m}$ is $4 \delta_{0}$-thin (see Remark 2.3), and then for any fixed $z \in \gamma_{m}^{*}$ there exists $z_{0}$ belonging to one of the other five sides of $H_{m}$ with $d_{\Omega}\left(z, z_{0}\right) \leq 4 \delta_{0}$. Let us analyze now what happens depending on what side of the fundamental hexagon $H_{m}$ the point $z_{0}$ belongs to:

Firstly, in the case that $z_{0} \in\left(a_{0}, b_{0}\right) \cup\left(a_{m}, b_{m}\right) \cup\left(a_{m+1}, b_{m+1}\right)$, then $d_{\Omega}(z, \mathbb{R})$ $\leq 4 \delta_{0}$.

Secondly, if $z_{0} \in \sigma_{m}$, then $d_{\Omega}\left(z_{0}, \mathbb{R}\right) \leq d_{\Omega}\left(z_{0},\left(a_{m}, b_{m}\right) \cup\left(a_{m+1}, b_{m+1}\right)\right) \leq$ $r_{m} \leq C$. Therefore, $d_{\Omega}(z, \mathbb{R}) \leq 4 \delta_{0}+C$.

Finally, if $z_{0} \in \gamma_{m+1}^{*}=\gamma_{n_{k+1}}^{*}$, then $d_{\Omega}(z, \mathbb{R}) \leq d_{\Omega}\left(z_{0},\left(a_{0}, b_{0}\right)\right) \leq l_{n_{k+1}} \leq c_{0}$. Hence, $d_{\Omega}(z, \mathbb{R}) \leq 4 \delta_{0}+c_{0}$.

Consequently, taking into account every possible case, $d_{\Omega}(z, \mathbb{R}) \leq 4 \delta_{0}+$ $\max \left\{c_{0}, C\right\}$. Applying now Theorem 2.5 we conclude that $\Omega$ is hyperbolic.

In order to prove the converse, let us assume now that

$$
\sup _{k}\left\{\min \left\{l_{n_{k}+1}, r_{n_{k}}, r_{n_{k}+1}\right\}: n_{k+1}-n_{k}=2\right\}=\infty .
$$

It means that there exists a subsequence $\left\{m_{j}\right\}_{j} \subseteq\left\{n_{k}\right\}_{k}$ such that

$$
\lim _{j \rightarrow \infty} \min \left\{l_{m_{j}+1}, r_{m_{j}}, r_{m_{j}+1}\right\}=\infty .
$$

That is to say, $\lim _{j \rightarrow \infty} l_{m_{j}+1}=\lim _{j \rightarrow \infty} r_{m_{j}}=\lim _{j \rightarrow \infty} r_{m_{j}+1}=\infty$. Let us fix now $m_{j}$; there exists $k$ such that $m_{j}=n_{k}$ and therefore $m_{j}+2=n_{k+1}$. Then, $l_{m_{j}}, l_{m_{j}+2} \leq c_{0}$ and, obviously, $l_{m_{j}}, l_{m_{j}+2} \leq l_{m_{j}+1}+c_{0}$. Theorem 2.8 gives the final argument to finish the proof. 
Next, a sufficient condition for hyperbolicity.

Theorem 3.2. Let $\Omega$ be a Denjoy domain. If there exists $C$ such that $r_{n} \leq C$ for every $n$ and there exist $N$ and $\left\{n_{k}\right\}_{k}$ such that $1 \leq n_{k+1}-n_{k} \leq N$ and $\sup _{k} l_{n_{k}}<\infty$, then $\Omega$ is hyperbolic.

Proof. Notice that there are, at most, $N-1$ fundamental geodesics with unbounded length between every two consecutive elements in the subsequence $\left\{\gamma_{n_{k}}^{*}\right\}_{k}$, and that by hypothesis, the lengths $l_{n_{k}}$ of the fundamental geodesics $\gamma_{n_{k}}^{*}$ in the subsequence verify that $l_{n_{k}} \leq c_{0}$ for some constant $c_{0}$.

In order to apply Theorem 2.5 , we have to show that $d_{\Omega}(z, \mathbb{R}) \leq c$ for some constant $c$ and every $z \in \cup_{m} \gamma_{m}^{*}$.

We claim first that $d_{\Omega}(z, \mathbb{R}) \leq c_{j}$ for some constant $c_{j}$ and every $z \in \gamma_{n_{k}+j}^{*}$, with $0 \leq j<n_{k+1}-n_{k}$. We will prove this claim by induction on $j$, with $0 \leq j<N$.

Notice that, if $j=0$, then $z$ belongs to $\gamma_{n_{k}}^{*}$, one of the fundamental geodesics that verify $l_{n_{k}} \leq c_{0}$, and then $d_{\Omega}(z, \mathbb{R}) \leq d_{\Omega}\left(z,\left(a_{0}, b_{0}\right)\right) \leq l_{n_{k}} \leq c_{0}$. Let us assume now that the claim is true for $j-1$ and let us prove it for $j$.

We know that the fundamental hexagon $H$ with two of its sides coincident with the fundamental geodesic $\gamma_{n_{k}+j}^{*}$ and the previous one, $\gamma_{n_{k}+j-1}^{*}$, is $4 \delta_{0^{-}}$ thin, (see Remark 2.3). It means that for any fixed $z \in \gamma_{n_{k}+j}^{*}$ there exists $z_{0}$ belonging to one of the other five sides of $H$ with $d_{\Omega}\left(z, z_{0}\right) \leq 4 \delta_{0}$. Repeating the argument in the proof of Theorem 3.1 and analyzing what happens depending on what side of the fundamental hexagon $H$ the point $z_{0}$ belongs to, we can easily see that the worst case scenario occurs when $z_{0} \in \gamma_{n_{k}+j-1}^{*}$ and in that case, by inductive assumption, $d_{\Omega}(z, \mathbb{R}) \leq 4 \delta_{0}+c_{j-1}$. Otherwise, $d_{\Omega}(z, \mathbb{R}) \leq$ $4 \delta_{0}+C$. That is to say, for every $z \in \gamma_{n_{k}+j}^{*}$, we have that $d_{\Omega}(z, \mathbb{R}) \leq 4 \delta_{0}+$ $\max \left\{c_{j-1}, C\right\}$. Iterating the argument $j-1$ additional times (and taking into account that $0 \leq j<N$ ) on the fundamental hexagons located to the left of $H$, we can conclude that $d_{\Omega}(z, \mathbb{R}) \leq 4(N-1) \delta_{0}+\max \left\{c_{0}, C\right\}$ for every $z \in \gamma_{n_{k}+j}^{*}$ with $0 \leq j<N$. Consequently, $d_{\Omega}(z, \mathbb{R}) \leq 4(N-1) \delta_{0}+\max \left\{c_{0}, C\right\}$ for every $z \in \cup_{m} \gamma_{m}^{*}$.

Theorem 2.5 gives the final argument to finish the proof.

Theorem 3.9 gives sufficient conditions for non-hyperbolicity. In order to simplify its proof, we will split it into several lemmas.

Lemma 3.3. Let $\Omega$ be a train such that there exists a subsequence $\left\{n_{k}\right\}_{k}$ of natural numbers and a constant $c$ with $l_{n_{k}}, l_{n_{k}+3} \leq c$ for every $k$. Assume also that $r_{n_{k}}, l_{n_{k}+1}-l_{n_{k}+2} \leq c$ for every $k$ and $\lim _{k \rightarrow \infty} r_{n_{k}+1}=\lim _{k \rightarrow \infty} r_{n_{k}+2}=$ $\lim _{k \rightarrow \infty} l_{n_{k}+2}=\infty$. Then $\Omega$ is not hyperbolic.

Proof. The conclusion is straightforward applying Theorem 2.8.

Lemma 3.4. Let us consider a train $\Omega$ and some fixed $n$. We take $z \in \gamma_{n}^{*}$ with $h(z)=l_{n}-s$. Then

$$
d_{\Omega}\left(z,\left(a_{0}, b_{0}\right)\right)=l_{n}-s, \quad d_{\Omega}\left(z,\left(a_{n+1}, b_{n+1}\right)\right) \geq r_{n}-s,
$$




$$
d_{\Omega}\left(z, \gamma_{n+1}^{*}\right) \geq \operatorname{Arcsinh} e^{\frac{1}{2}\left(r_{n}+l_{n}-l_{n+1}-2 s\right)} .
$$

Proof. It is straightforward that $d_{\Omega}\left(z_{n},\left(a_{0}, b_{0}\right)\right)=h\left(z_{n}\right)=l_{n}-s$. We also have that $r_{n}=d_{\Omega}\left(\left(a_{n}, b_{n}\right),\left(a_{n+1}, b_{n+1}\right)\right) \leq s+d_{\Omega}\left(z_{n},\left(a_{n}, b_{n}\right)\right)$, and then

$$
d_{\Omega}\left(z_{n},\left(a_{n}, b_{n}\right)\right) \geq r_{n}-s .
$$

Standard hyperbolic trigonometry (see e.g. [9, p. 161]) in $H_{n}$ (see Definition 2.7 in order to recall the definitions of $\left.H_{n}, \alpha_{n}, \ldots\right)$ gives

$$
\cosh \alpha_{n}=\frac{\cosh r_{n}+\cosh l_{n} \cosh l_{n+1}}{\sinh l_{n} \sinh l_{n+1}} \geq \frac{\frac{1}{2} e^{r_{n}}}{\frac{1}{2} e^{l_{n}} \frac{1}{2} e^{l_{n+1}}}+1=1+2 e^{r_{n}-l_{n}-l_{n+1}} .
$$

Then, we have

$$
\frac{1}{2} \sinh \alpha_{n} \geq \sinh \frac{\alpha_{n}}{2}=\sqrt{\frac{\cosh \alpha_{n}-1}{2}} \geq e^{\frac{1}{2}\left(r_{n}-l_{n}-l_{n+1}\right)} .
$$

Standard hyperbolic trigonometry for right-angled quadrilaterals (see e.g. [9, p. 157]) gives

$$
\begin{aligned}
\sinh d_{\Omega}\left(z_{n}, \gamma_{n+1}^{*}\right) & =\sinh \alpha_{n} \cosh \left(l_{n}-s\right) \\
& \geq 2 e^{\frac{1}{2}\left(r_{n}-l_{n}-l_{n+1}\right)} \frac{1}{2} e^{l_{n}-s}=e^{\frac{1}{2}\left(r_{n}+l_{n}-l_{n+1}-2 s\right)} .
\end{aligned}
$$

Lemma 3.5. Let $\Omega$ be a train such that there exists a subsequence $\left\{n_{k}\right\}_{k}$ of natural numbers and a constant $c$ with $l_{n_{k}}, l_{n_{k}+3} \leq c$ for every $k$. Assume also that $r_{n_{k}}, l_{n_{k}+1}+l_{n_{k}+2}-r_{n_{k}+1} \leq c$ for every $k$ and $\lim _{k \rightarrow \infty} r_{n_{k}+1}=$ $\lim _{k \rightarrow \infty} r_{n_{k}+2}=\lim _{k \rightarrow \infty} l_{n_{k}+2}=\lim _{k \rightarrow \infty}\left(l_{n_{k}+1}-l_{n_{k}+2}\right)=\infty$. Then $\Omega$ is not hyperbolic.

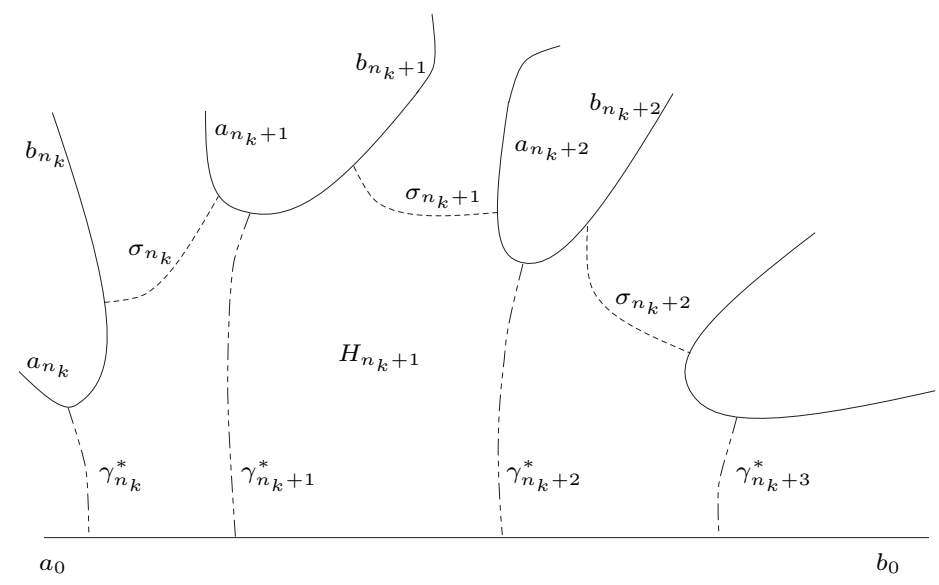


Proof. Consider $z_{k} \in \gamma_{n_{k}+2}^{*}$ with height $h\left(z_{k}\right)=l_{n_{k}+2}-s_{k}$, where $s_{k}:=$ $\log \left(\min \left\{l_{n_{k}+2}, r_{n_{k}+2}\right\}\right)$.

It is direct that $d_{\Omega}\left(z_{k},\left(a_{n_{k}+2}, b_{n_{k}+2}\right)\right)=s_{k} \longrightarrow \infty$, if $k \longrightarrow \infty$.

Lemma 3.4 implies the following facts:

$$
\begin{aligned}
d_{\Omega}\left(z_{k},\left(a_{0}, b_{0}\right)\right) & \geq l_{n_{k}+2}-\log l_{n_{k}+2} \longrightarrow \infty, \\
d_{\Omega}\left(z_{k},\left(a_{n_{k}+3}, b_{n_{k}+3}\right)\right) & \geq r_{n_{k}+2}-\log r_{n_{k}+2} \longrightarrow \infty, \\
d_{\Omega}\left(z_{k}, \gamma_{n_{k}+3}\right) & \geq \operatorname{Arcsinh} e^{\frac{1}{2}\left(r_{n_{k}+2}+l_{n_{k}+2}-l_{n_{k}+3}-2 \log r_{n_{k}+2}\right)} \\
& \geq \operatorname{Arcsinh} e^{\frac{1}{2}\left(r_{n_{k}+2}+l_{n_{k}+2}-c-2 \log r_{n_{k}+2}\right)} \longrightarrow \infty,
\end{aligned}
$$

if $k \rightarrow \infty$. Note that the geodesic joining $z_{k}$ and $\cup_{n>n_{k}+3}\left(a_{n}, b_{n}\right)$ must intersect $\gamma_{n_{k}+3}$ (see the figure above); hence,

$$
d_{\Omega}\left(z_{k}, \cup_{n>n_{k}+3}\left(a_{n}, b_{n}\right)\right) \geq d_{\Omega}\left(z_{k}, \gamma_{n_{k}+3}\right) \longrightarrow \infty,
$$

if $k \rightarrow \infty$. Then $\lim _{k \rightarrow \infty} d_{\Omega}\left(z_{k}, \cup_{n \geq n_{k}+2}\left(a_{n}, b_{n}\right) \cup\left(a_{0}, b_{0}\right)\right)=\infty$.

Since every $w \in\left(a_{n_{k}+1}, b_{n_{k}+1}\right)$ verifies $h(w):=d_{\Omega}\left(w,\left(a_{0}, b_{0}\right)\right) \geq l_{n_{k}+1}$, we obtain by Lemma 2.10

$$
d_{\Omega}\left(z_{k},\left(a_{n_{k}+1}, b_{n_{k}+1}\right)\right) \geq h(w)-h\left(z_{k}\right) \geq l_{n_{k}+1}-l_{n_{k}+2}+s_{k} \geq s_{k} \longrightarrow \infty,
$$

if $k \rightarrow \infty$.

Let us consider now the fundamental hexagon $H_{n_{k}+1}$ (see Definition 2.7). Standard hyperbolic trigonometry (see e.g. [9, p. 161]) gives

$$
\cosh \alpha_{n_{k}+1}=\frac{\cosh r_{n_{k}+1}+\cosh l_{n_{k}+1} \cosh l_{n_{k}+2}}{\sinh l_{n_{k}+1} \sinh l_{n_{k}+2}} \approx 1+2 e^{r_{n_{k}+1}-l_{n_{k}+1}-l_{n_{k}+2}},
$$

if $k \rightarrow \infty$. Since $l_{n_{k}+1}+l_{n_{k}+2}-r_{n_{k}+1} \leq c$, we deduce that $\alpha_{n_{k}+1} \geq c_{0}$ for some positive constant $c_{0}$. Standard hyperbolic trigonometry (see e.g. [9], p. 157) gives

$$
\begin{aligned}
\sinh d_{\Omega}\left(z_{k}, \gamma_{n_{k}+1}\right) & =\sinh \alpha_{n_{k}+1} \cosh h\left(z_{k}\right) \\
& \geq \sinh c_{0} \cosh \left(l_{n_{k}+2}-\log l_{n_{k}+2}\right) \longrightarrow \infty,
\end{aligned}
$$

if $k \rightarrow \infty$. Therefore,

$$
d_{\Omega}\left(z_{k}, \cup_{n=1}^{n_{k}}\left(a_{n}, b_{n}\right)\right) \geq d_{\Omega}\left(z_{k}, \gamma_{n_{k}+1}\right) \longrightarrow \infty,
$$

if $k \rightarrow \infty$, and we obtain $\lim _{k \rightarrow \infty} d_{\Omega}\left(z_{k}, \cup_{n=1}^{n_{k}+1}\left(a_{n}, b_{n}\right)\right)=\infty$.

Consequently, $\lim _{k \rightarrow \infty} d_{\Omega}\left(z_{k}, \mathbb{R}\right)=\infty$. Hence, $\Omega$ is not hyperbolic by Theorem 2.5 .

Lemma 3.6. Let $\Omega$ be a train such that there exists a subsequence $\left\{n_{k}\right\}_{k}$ of natural numbers and a constant $c$ with $l_{n_{k}}, l_{n_{k}+3} \leq c$ for every $k$. Assume that $r_{n_{k}} \leq c$ for every $k$ and $\lim _{k \rightarrow \infty} r_{n_{k}+1}=\lim _{k \rightarrow \infty} r_{n_{k}+2}=\lim _{k \rightarrow \infty} l_{n_{k}+2}=$ $\lim _{k \rightarrow \infty}\left(l_{n_{k}+1}-l_{n_{k}+2}\right)=\lim _{k \rightarrow \infty}\left(l_{n_{k}+1}+l_{n_{k}+2}-r_{n_{k}+1}\right)=\lim _{k \rightarrow \infty}\left(l_{n_{k}+2}-\right.$ $\left.l_{n_{k}+1}+r_{n_{k}+1}\right)=\infty$. Then $\Omega$ is not hyperbolic. 
Proof. Consider $z_{k} \in \gamma_{n_{k}+2}^{*}$ with height $h\left(z_{k}\right)=l_{n_{k}+2}-s_{k}$, where

$$
s_{k}:=\log \left(\min \left\{l_{n_{k}+2}, r_{n_{k}+2}, l_{n_{k}+2}-l_{n_{k}+1}+r_{n_{k}+1}\right\}\right) .
$$

Repeating exactly the argument in the proof of Lemma 3.5, we conclude that $\lim _{k \rightarrow \infty} d_{\Omega}\left(z_{k}, \cup_{n \geq n_{k}+1}\left(a_{n}, b_{n}\right) \cup\left(a_{0}, b_{0}\right)\right)=\infty$.

Let us consider now the fundamental hexagon $H_{n_{k}+1}$ (see Definition 2.7). Standard hyperbolic trigonometry (see e.g. [9, p. 161]) gives

$$
\cosh \alpha_{n_{k}+1}=\frac{\cosh r_{n_{k}+1}+\cosh l_{n_{k}+1} \cosh l_{n_{k}+2}}{\sinh l_{n_{k}+1} \sinh l_{n_{k}+2}} \approx 1+2 e^{r_{n_{k}+1}-l_{n_{k}+1}-l_{n_{k}+2}},
$$

if $k \rightarrow \infty$. Since $\lim _{k \rightarrow \infty}\left(l_{n_{k}+1}+l_{n_{k}+2}-r_{n_{k}+1}\right)=\infty$, we deduce that

$$
\begin{aligned}
\cosh \alpha_{n_{k}+1} & \approx 1+\frac{1}{2} \alpha_{n_{k}+1}^{2} \approx 1+2 e^{r_{n_{k}+1}-l_{n_{k}+1}-l_{n_{k}+2}}, \\
\alpha_{n_{k}+1} & \approx 2 e^{\frac{1}{2}\left(-l_{n_{k}+1}-l_{n_{k}+2}+r_{n_{k}+1}\right)},
\end{aligned}
$$

if $k \longrightarrow \infty$. Standard hyperbolic trigonometry (see e.g. [9, p. 157]) gives

$$
\begin{aligned}
\sinh d_{\Omega}\left(z_{k}, \gamma_{n_{k}+1}\right) & =\sinh \alpha_{n_{k}+1} \cosh h\left(z_{k}\right) \\
& \geq c 2 e^{\frac{1}{2}\left(-l_{n_{k}+1}-l_{n_{k}+2}+r_{n_{k}+1}\right)} \frac{1}{2} e^{l_{n_{k}+2}-s_{k}} \\
& \geq c e^{\frac{1}{2}\left(l_{n_{k}+2}-l_{n_{k}+1}+r_{n_{k}}+1\right)-\log \left(l_{n_{k}+2}-l_{n_{k}+1}+r_{n_{k}+1}\right)} \longrightarrow \infty,
\end{aligned}
$$

if $k \rightarrow \infty$. Therefore,

$$
d_{\Omega}\left(z_{k}, \cup_{n=1}^{n_{k}}\left(a_{n}, b_{n}\right)\right) \geq d_{\Omega}\left(z_{k}, \gamma_{n_{k}+1}\right) \longrightarrow \infty,
$$

if $k \rightarrow \infty$, and we obtain $\lim _{k \rightarrow \infty} d_{\Omega}\left(z_{k}, \mathbb{R}\right)=\infty$. Hence, $\Omega$ is not hyperbolic by Theorem 2.5 .

Lemma 3.7. Let $\Omega$ be a train such that there exists a subsequence $\left\{n_{k}\right\}_{k}$ of natural numbers and a constant $c$ with $l_{n_{k}}, l_{n_{k}+3} \leq c$ for every $k$. Assume that $r_{n_{k}+1}, l_{n_{k}+2}-l_{n_{k}+1} \leq c$ for every $k$, and $\lim _{k \rightarrow \infty} r_{n_{k}}=\lim _{k \rightarrow \infty} r_{n_{k}+2}=$ $\lim _{k \rightarrow \infty} l_{n_{k}+2}=\lim _{k \rightarrow \infty}\left(l_{n_{k}+2}-l_{n_{k}+1}+r_{n_{k}}\right)=\infty$. Then $\Omega$ is not hyperbolic.

Note that, under these hypotheses, we have $\lim _{k \rightarrow \infty} l_{n_{k}+1}=\infty$.

Proof. Consider $z_{k} \in \gamma_{n_{k}+2}^{*}$ with height $h\left(z_{k}\right)=l_{n_{k}+2}-s_{k}$, where

$$
s_{k}:=\log \left(\min \left\{l_{n_{k}+2}, r_{n_{k}+2}, l_{n_{k}+2}-l_{n_{k}+1}+r_{n_{k}}\right\}\right) \text {. }
$$

Repeating exactly the argument in the proof of Lemma 3.5, we conclude that $\lim _{k \rightarrow \infty} d_{\Omega}\left(z_{k}, \cup_{n \geq n_{k}+2}\left(a_{n}, b_{n}\right) \cup\left(a_{0}, b_{0}\right)\right)=\infty$.

If $k$ is large enough, when $w$ is the nearest point in $\left(a_{n_{k}+1}, b_{n_{k}+1}\right)$ to $z_{k}$, by Lemma 2.10,

$$
d_{\Omega}\left(z_{k},\left(a_{n_{k}+1}, b_{n_{k}+1}\right)\right) \geq h(w)-h\left(z_{k}\right) \geq l_{n_{k}+1}-l_{n_{k}+2}+s_{k} \geq s_{k}-c \longrightarrow \infty,
$$

if $k \rightarrow \infty$.

Seeking for a contradiction let us assume that $\Omega$ is hyperbolic. Then, by Theorem 2.5, there exists a constant $c_{0}$ such that $d_{\Omega}\left(z_{k}, \mathbb{R}\right) \leq c_{0}$ for every $k$. Since $\lim _{k \rightarrow \infty} h\left(z_{k}\right)=\infty$ and $l_{n_{k}} \leq c$, then $d_{\Omega}\left(z_{k},\left(a_{n_{k}}, b_{n_{k}}\right)\right) \leq c_{0}$ for $k$ large enough. 
Let us denote by $w_{k}$ the point in $\left(a_{n_{k}}, b_{n_{k}}\right)$ with $d_{\Omega}\left(z_{k},\left(a_{n_{k}}, b_{n_{k}}\right)\right)=d_{\Omega}\left(z_{k}, w_{k}\right)$. Consider the point $z_{k}^{\prime} \in \gamma_{n_{k}+1}^{*}$ with $d_{\Omega}\left(z_{k}, w_{k}\right)=d_{\Omega}\left(z_{k}, z_{k}^{\prime}\right)+d_{\Omega}\left(z_{k}^{\prime}, w_{k}\right) \leq c_{0}$. By Lemma 2.10, we conclude that $\left|h\left(z_{k}^{\prime}\right)-h\left(z_{k}\right)\right| \leq d_{\Omega}\left(z_{k}, z_{k}^{\prime}\right) \leq c_{0}$. Since

$$
\begin{aligned}
r_{n_{k}}=d_{\Omega}\left(\left(a_{n_{k}}, b_{n_{k}}\right),\left(a_{n_{k}+1}, b_{n_{k}+1}\right)\right) & \leq d_{\Omega}\left(z_{k}^{\prime}, w_{k}\right)+l_{n_{k}+1}-h\left(z_{k}^{\prime}\right) \\
& \leq c_{0}+l_{n_{k}+1}-h\left(z_{k}\right)+c_{0} .
\end{aligned}
$$

We conclude that $r_{n_{k}}-l_{n_{k}+1}+l_{n_{k}+2}-s_{k} \leq 2 c_{0}$. Then,

$$
l_{n_{k}+2}-l_{n_{k}+1}+r_{n_{k}}-\log \left(l_{n_{k}+2}-l_{n_{k}+1}+r_{n_{k}}\right) \leq 2 c_{0},
$$

and this contradicts the assumption $\lim _{k \rightarrow \infty}\left(l_{n_{k}+2}-l_{n_{k}+1}+r_{n_{k}}\right)=\infty$. Therefore $\Omega$ is not hyperbolic.

Lemma 3.8. Let $\Omega$ be a train such that there exists a subsequence $\left\{n_{k}\right\}_{k}$ of natural numbers and a constant $c$ with $l_{n_{k}}, l_{n_{k}+3} \leq c$ for every $k$. Assume also that $\lim _{k \rightarrow \infty} r_{n_{k}}=\lim _{k \rightarrow \infty} r_{n_{k}+1}=\lim _{k \rightarrow \infty} r_{n_{k}+2}=\lim _{k \rightarrow \infty} \max \left\{l_{n_{k}+1}, l_{n_{k}+2}\right\}=$ $\infty$. Then $\Omega$ is not hyperbolic.

Proof. We define a sequence of natural numbers $\left\{m_{k}\right\}_{k}$ in the following way

$$
m_{k}:= \begin{cases}n_{k}+1 & \text { if } l_{n_{k}+1} \geq l_{n_{k}+2}, \\ n_{k}+2 & \text { if } l_{n_{k}+2} \geq l_{n_{k}+1},\end{cases}
$$

with $m_{1}$ large enough so that $l_{m_{k}} \geq c$ for every $k$. Then, $\lim _{k \rightarrow \infty} l_{m_{k}}=$ $\infty, l_{m_{k}} \geq l_{m_{k}-1}, l_{m_{k}+1}$ and $\lim _{k \rightarrow \infty} r_{m_{k}-1}=\lim _{k \rightarrow \infty} r_{m_{k}}=\infty$. Applying Theorem 2.8 to $\left\{m_{k}\right\}_{k}$, we conclude that $\Omega$ is not hyperbolic.

In the two following theorems the items labeled with $\left(j^{\prime}\right)$ cover the symmetric case to the one listed exactly one position above (i.e., the one labeled with $(j)$ ).

Lemmas 3.3-3.8 give directly the following result for non-hyperbolicity.

Theorem 3.9. Let $\Omega$ be a train such that there exists a subsequence $\left\{n_{k}\right\}_{k}$ of natural numbers and a constant $c$ with $l_{n_{k}}, l_{n_{k}+3} \leq c$ for every $k$. Then $\Omega$ is not hyperbolic if we have either:

(a) $\sup _{k} r_{n_{k}}<\infty$,

$$
\begin{aligned}
& \lim _{k \rightarrow \infty} r_{n_{k}+1}=\lim _{k \rightarrow \infty} r_{n_{k}+2}=\lim _{k \rightarrow \infty} l_{n_{k}+2}=\infty \quad \text { and } \\
& \sup _{k}\left(l_{n_{k}+1}-l_{n_{k}+2}\right)<\infty
\end{aligned}
$$

(a') $\sup _{k} r_{n_{k}+2}<\infty$,

$$
\begin{aligned}
& \lim _{k \rightarrow \infty} r_{n_{k}}=\lim _{k \rightarrow \infty} r_{n_{k}+1}=\lim _{k \rightarrow \infty} l_{n_{k}+1}=\infty \quad \text { and } \\
& \sup _{k}\left(l_{n_{k}+2}-l_{n_{k}+1}\right)<\infty
\end{aligned}
$$

(b) $\sup _{k} r_{n_{k}}<\infty$,

$$
\begin{aligned}
& \lim _{k \rightarrow \infty} r_{n_{k}+1}=\lim _{k \rightarrow \infty} r_{n_{k}+2}=\lim _{k \rightarrow \infty} l_{n_{k}+2}=\lim _{k \rightarrow \infty}\left(l_{n_{k}+1}-l_{n_{k}+2}\right)=\infty \quad \text { and } \\
& \sup _{k}\left(l_{n_{k}+1}+l_{n_{k}+2}-r_{n_{k}+1}\right)<\infty .
\end{aligned}
$$


(b') $\sup _{k} r_{n_{k}+2}<\infty$,

$$
\begin{aligned}
& \lim _{k \rightarrow \infty} r_{n_{k}}=\lim _{k \rightarrow \infty} r_{n_{k}+1}=\lim _{k \rightarrow \infty} l_{n_{k}+1}=\lim _{k \rightarrow \infty}\left(l_{n_{k}+2}-l_{n_{k}+1}\right)=\infty \quad \text { and } \\
& \sup _{k}\left(l_{n_{k}+1}+l_{n_{k}+2}-r_{n_{k}+1}\right)<\infty . \\
& \begin{aligned}
& \text { (c) } \sup _{k} r_{n_{k}}<\infty \text { and } \\
& \lim _{k \rightarrow \infty} r_{n_{k}+1}=\lim _{k \rightarrow \infty} r_{n_{k}+2}=\lim _{k \rightarrow \infty} l_{n_{k}+2}=\lim _{k \rightarrow \infty}\left(l_{n_{k}+1}-l_{n_{k}+2}\right) \\
&=\lim _{k \rightarrow \infty}\left(l_{n_{k}+1}+l_{n_{k}+2}-r_{n_{k}+1}\right)=\lim _{k \rightarrow \infty}\left(l_{n_{k}+2}-l_{n_{k}+1}+r_{n_{k}+1}\right) \\
&=\infty .
\end{aligned}
\end{aligned}
$$

(c') $\sup _{k} r_{n_{k}+2}<\infty$ and

$$
\begin{aligned}
\lim _{k \rightarrow \infty} r_{n_{k}} & =\lim _{k \rightarrow \infty} r_{n_{k}+1}=\lim _{k \rightarrow \infty} l_{n_{k}+1}=\lim _{k \rightarrow \infty}\left(l_{n_{k}+2}-l_{n_{k}+1}\right) \\
& =\lim _{k \rightarrow \infty}\left(l_{n_{k}+1}+l_{n_{k}+2}-r_{n_{k}+1}\right)=\lim _{k \rightarrow \infty}\left(l_{n_{k}+1}-l_{n_{k}+2}+r_{n_{k}+1}\right)=\infty .
\end{aligned}
$$

(d) $\sup _{k} r_{n_{k}+1}<\infty, \sup _{k}\left(l_{n_{k}+2}-l_{n_{k}+1}\right)<\infty$ and

$\lim _{k \rightarrow \infty} r_{n_{k}}=\lim _{k \rightarrow \infty} r_{n_{k}+2}=\lim _{k \rightarrow \infty} l_{n_{k}+2}=\lim _{k \rightarrow \infty}\left(l_{n_{k}+2}-l_{n_{k}+1}+r_{n_{k}}\right)=\infty$.

(d') $\sup _{k} r_{n_{k}+1}<\infty, \sup _{k}\left(l_{n_{k}+1}-l_{n_{k}+2}\right)<\infty$ and

$\lim _{k \rightarrow \infty} r_{n_{k}}=\lim _{k \rightarrow \infty} r_{n_{k}+2}=\lim _{k \rightarrow \infty} l_{n_{k}+1}=\lim _{k \rightarrow \infty}\left(l_{n_{k}+1}-l_{n_{k}+2}+r_{n_{k}+2}\right)=\infty$.

(e) $\lim _{k \rightarrow \infty} r_{n_{k}}=\lim _{k \rightarrow \infty} r_{n_{k}+1}=\lim _{k \rightarrow \infty} r_{n_{k}+2}$

$$
=\lim _{k \rightarrow \infty} \max \left\{l_{n_{k}+1}, l_{n_{k}+2}\right\}=\infty .
$$

We prove now the following sufficient condition for hyperbolicity.

Theorem 3.10. Let $\Omega$ be a train such that there exists a subsequence $S:=$ $\left\{n_{k}\right\}_{k}$ with $n_{k+1}-n_{k}=3$ and $\sup _{k} l_{n_{k}}<\infty$. Then $\Omega$ is hyperbolic if there exist a constant $C$ and a partition $\left\{A_{1}, A_{2}, A_{3}, A_{4}, A_{5}, A_{6}, A_{7}, A_{8}\right\}$ of $\mathbb{N}$ such that each $A_{j}$ is either empty or infinite and all of the following conditions hold:

(a) $\min \left\{\max \left\{r_{n_{k}}, r_{n_{k}+1}\right\}, \max \left\{r_{n_{k}}, r_{n_{k}+2}\right\}, \max \left\{r_{n_{k}+1}, r_{n_{k}+2}\right\}\right\} \leq C$ for every $k \in A_{1}$.

(b) $\min \left\{\max \left\{r_{n_{k}}, l_{n_{k}+2}\right\}, \max \left\{r_{n_{k}+2}, l_{n_{k}+1}\right\}\right\} \leq C$ for every $k \in A_{2}$.

(c) $\max \left\{r_{n_{k}}, l_{n_{k}+2}-l_{n_{k}+1}+r_{n_{k}+1}\right\} \leq C$ for every $k \in A_{3}$, and

$$
\begin{aligned}
\lim _{k \rightarrow \infty, k \in A_{3}} r_{n_{k}+1} & =\lim _{k \rightarrow \infty, k \in A_{3}} l_{n_{k}+2}=\lim _{k \rightarrow \infty, k \in A_{3}}\left(l_{n_{k}+1}-l_{n_{k}+2}\right) \\
& =\lim _{k \rightarrow \infty, k \in A_{3}}\left(l_{n_{k}+1}+l_{n_{k}+2}-r_{n_{k}+1}\right)=\infty .
\end{aligned}
$$

(c') $\max \left\{r_{n_{k}+2}, l_{n_{k}+1}-l_{n_{k}+2}+r_{n_{k}+1}\right\} \leq C$ for every $k \in A_{4}$, and

$$
\begin{aligned}
\lim _{k \rightarrow \infty, k \in A_{4}} r_{n_{k}+1} & =\lim _{k \rightarrow \infty, k \in A_{4}} l_{n_{k}+1}=\lim _{k \rightarrow \infty, k \in A_{4}}\left(l_{n_{k}+2}-l_{n_{k}+1}\right) \\
& =\lim _{k \rightarrow \infty, k \in A_{4}}\left(l_{n_{k}+1}+l_{n_{k}+2}-r_{n_{k}+1}\right)=\infty
\end{aligned}
$$


(d) $\max \left\{r_{n_{k}+1}, \min \left\{l_{n_{k}+1}, l_{n_{k}+2}\right\}\right\} \leq C$ for every $k \in A_{5}$.

(e) $\max \left\{r_{n_{k}+1}, l_{n_{k}+2}-l_{n_{k}+1}+r_{n_{k}}\right\} \leq C$ for every $k \in A_{6}$, and

$$
\lim _{k \rightarrow \infty, k \in A_{6}} l_{n_{k}+1}=\lim _{k \rightarrow \infty, k \in A_{6}} l_{n_{k}+2}=\infty .
$$

(e') $\max \left\{r_{n_{k}+1}, l_{n_{k}+1}-l_{n_{k}+2}+r_{n_{k}+2}\right\} \leq C$ for every $k \in A_{7}$, and

$$
\lim _{k \rightarrow \infty, k \in A_{7}} l_{n_{k}+1}=\lim _{k \rightarrow \infty, k \in A_{7}} l_{n_{k}+2}=\infty .
$$

(f) $\max \left\{l_{n_{k}+1}, l_{n_{k}+2}\right\} \leq C$ for every $k \in A_{8}$.

Proof. First of all notice that, by hypothesis, the lengths $l_{n_{k}}$ of the fundamental geodesics $\gamma_{n_{k}}^{*}$ belonging to the subsequence $S$ satisfy $l_{n_{k}} \leq c$ for some constant $c$. Therefore, if $m \in\left\{n_{k}\right\}_{k}$, then $d_{\Omega}(z, \mathbb{R}) \leq l_{m} \leq c$ for every $z \in \gamma_{m}^{*}$. It means that we just have to care about $d_{\Omega}(z, \mathbb{R})$ for every $z \in\left\{\gamma_{m}^{*}\right\}_{m \notin S}$.

Assume first that $k \in A_{1}$. Let us consider $z \in \gamma_{n_{k}+1}^{*} \cup \gamma_{n_{k}+2}^{*}$ for some fixed $k \in A_{1}$. Let us assume that for instance, $r_{n_{k}}, r_{n_{k}+1} \leq C$, since the reasoning in any of the remaining cases in (a) is similar. If that is the situation, as we proved in Theorem 3.1, the fundamental hexagons $H_{n_{k}}$ and $H_{n_{k}+1}$ are $4 \delta_{0}$-thin and it means (repeating the same argument in that theorem) that $d_{\Omega}(z, \mathbb{R}) \leq 8 \delta_{0}+C$ for every $z \in \gamma_{n_{k}+1}^{*} \cup \gamma_{n_{k}+2}^{*}$.

Assume that $k \in A_{2}$. By symmetry, we can assume that $r_{n_{k}}, l_{n_{k}+2} \leq C$. Since the fundamental hexagon $H_{n_{k}}$ is $4 \delta_{0}$-thin, we know that $d_{\Omega}(z, \mathbb{R}) \leq$ $4 \delta_{0}+C$ for every $z \in \gamma_{n_{k}+1}^{*}$. We also have $d_{\Omega}(z, \mathbb{R}) \leq l_{n_{k}+2} \leq C$ for every $z \in \gamma_{n_{k}+2}^{*}$.

Consider $k \in A_{3}$. Note that the fundamental hexagon $H_{n_{k}}$ is $4 \delta_{0}$-thin and it means that $d_{\Omega}(z, \mathbb{R}) \leq 4 \delta_{0}+C$ for every $z \in \cup_{k \in A_{3}} \gamma_{n_{k}+1}^{*}$.

Consider $z \in \gamma_{n_{k}+2}^{*}$ with height $h(z)=h \in\left[0, l_{n_{k}+2}\right]$.

Let us consider now the fundamental hexagon $H_{n_{k}+1}$. Standard hyperbolic trigonometry (see e.g. [9, p. 161]) gives

$$
\cosh \alpha_{n_{k}+1}=\frac{\cosh r_{n_{k}+1}+\cosh l_{n_{k}+1} \cosh l_{n_{k}+2}}{\sinh l_{n_{k}+1} \sinh l_{n_{k}+2}} \approx 1+2 e^{r_{n_{k}+1}-l_{n_{k}+1}-l_{n_{k}+2}},
$$

if $k \rightarrow \infty, k \in A_{3}$. Since $\lim _{k \rightarrow \infty, k \in A_{3}}\left(l_{n_{k}+1}+l_{n_{k}+2}-r_{n_{k}+1}\right)=\infty$, we deduce that

$$
\begin{aligned}
\cosh \alpha_{n_{k}+1} & \approx 1+\frac{1}{2} \alpha_{n_{k}+1}^{2} \approx 1+2 e^{r_{n_{k}+1}-l_{n_{k}+1}-l_{n_{k}+2}}, \\
\alpha_{n_{k}+1} & \approx 2 e^{-\frac{1}{2}\left(l_{n_{k}+1}+l_{n_{k}+2}-r_{n_{k}+1}\right)} .
\end{aligned}
$$

Standard hyperbolic trigonometry (see e.g. [9, p. 157]) gives

$$
\begin{aligned}
\sinh d_{\Omega}\left(z_{k}, \gamma_{n_{k}+1}\right) & =\sinh \alpha_{n_{k}+1} \cosh h \\
& \leq c e^{-\frac{1}{2}\left(l_{n_{k}+1}+l_{n_{k}+2}-r_{n_{k}+1}\right)+h} \\
& \leq c e^{-\frac{1}{2}\left(l_{n_{k}+1}+l_{n_{k}+2}-r_{n_{k}+1}\right)+l_{n_{k}+2}}
\end{aligned}
$$




$$
\leq c e^{\frac{1}{2}\left(l_{n_{k}+2}-l_{n_{k}+1}+r_{n_{k}+1}\right)} \leq c e^{C},
$$

if $k \in A_{3}$. Therefore,

$$
d_{\Omega}(z, \mathbb{R}) \leq 4 \delta_{0}+C+d_{\Omega}\left(z, \gamma_{n_{k}+1}\right) \leq 4 \delta_{0}+C+\operatorname{Arcsinh}\left(c e^{C}\right)
$$

for every $z \in \cup_{k \in A_{3}} \gamma_{n_{k}+2}^{*}$.

If $k \in A_{4}$, then we can use a symmetric argument to the previous one.

Assume now that $k \in A_{5}$. We have $r_{n_{k}+1} \leq C$ and $\min \left\{l_{n_{k}+1}, l_{n_{k}+2}\right\} \leq C$. By symmetry, we can assume that $l_{n_{k}+1} \leq C$. Then we have $d_{\Omega}(z, \mathbb{R}) \leq$ $l_{n_{k}+1} \leq C$ for every $z \in \gamma_{n_{k}+1}^{*}$.

Since the fundamental hexagon $H_{n_{k}+1}$ is $4 \delta_{0}$-thin, we have that $d_{\Omega}(z, \mathbb{R}) \leq$ $4 \delta_{0}+C$ for every $z \in \gamma_{n_{k}+2}^{*}$.

Let us consider $k \in A_{6}$.

Let $z \in \gamma_{n_{k}+1}^{*}$ with height $h(z)=h \in\left[0, l_{n_{k}+1}\right]$. If $h \in\left[0, l_{n_{k}}\right]$, then $d_{\Omega}(z, \mathbb{R}) \leq d_{\Omega}\left(z,\left(a_{0}, b_{0}\right)\right)=h \leq l_{n_{k}} \leq c$

Assume first that $l_{n_{k}+2} \leq l_{n_{k}+1}$. If $h \in\left[l_{n_{k}}, l_{n_{k}+2}\right]$, by Lemma 2.11,

$$
\begin{aligned}
d_{\Omega}\left(z,\left(a_{n_{k}}, b_{n_{k}}\right)\right) & \asymp e^{-h+l_{n_{k}}}+e^{-\left(l_{n_{k}+1}-h-r_{n_{k}}\right)_{+}}+\left(r_{n_{k}}+h-l_{n_{k}+1}\right)_{+} \\
& \leq 1+1+\left(r_{n_{k}}+l_{n_{k}+2}-l_{n_{k}+1}\right)_{+} \leq 2+C .
\end{aligned}
$$

If $h \in\left[l_{n_{k}+2}, l_{n_{k}+1}\right]$, then Lemma 2.12 gives that $d_{\Omega}\left(z,\left(a_{n_{k}+2}, b_{n_{k}+2}\right)\right) \leq c_{1}$, where $c_{1}$ only depends on $C$ and $\min _{k \in A_{6}} l_{n_{k}+2}>0$.

Assume now that $l_{n_{k}+1}<l_{n_{k}+2}$. If $h \in\left[l_{n_{k}}, l_{n_{k}+1}\right]$, by Lemma 2.11,

$$
\begin{aligned}
d_{\Omega}\left(z,\left(a_{n_{k}}, b_{n_{k}}\right)\right) & \asymp e^{-h+l_{n_{k}}}+e^{-\left(l_{n_{k}+1}-h-r_{n_{k}}\right)+}+\left(r_{n_{k}}+h-l_{n_{k}+1}\right)_{+} \\
& \leq 1+1+\left(r_{n_{k}}+l_{n_{k}+2}-l_{n_{k}+1}\right)_{+} \leq 2+C .
\end{aligned}
$$

Then we also have $d_{\Omega}\left(z,\left(a_{n_{k}+2}, b_{n_{k}+2}\right)\right) \leq c_{1}$.

Hence, $d_{\Omega}(z, \mathbb{R}) \leq c_{2}$ for every $z \in \gamma_{n_{k}+1}^{*}$.

If $z \in \gamma_{n_{k}+2}^{*}$, then $d_{\Omega}(z, \mathbb{R}) \leq 4 \delta_{0}+\max \left\{C, c_{2}\right\}$, since the fundamental hexagon $H_{n_{k}+1}$ is $4 \delta_{0}$-thin.

If $k \in A_{7}$, then we can use a symmetric argument to the previous one.

Finally, let us consider $k \in A_{8}$. For every $z \in \gamma_{n_{k}+1}^{*} \cup \gamma_{n_{k}+2}^{*}$ we have that $d_{\Omega}(z, \mathbb{R}) \leq d_{\Omega}\left(z,\left(a_{0}, b_{0}\right)\right) \leq \max \left\{l_{n_{k}+1}, l_{n_{k}+2}\right\} \leq C$.

This finishes the proof.

\section{A characterization for regular domains}

In this section we take advantage of the results proved in the previous section to deduce a characterization of hyperbolicity for some especially regular Denjoy domains.

Theorem 4.3 shows that the hypotheses in Theorems 3.9 and 3.10 are quite close to be complementary. 
Definition 4.1. We say that a sequence (or subsequence) is regular if either it is upper bounded or its limit is infinity. Let $\Omega$ be a train such that there exists a subsequence $\left\{n_{k}\right\}_{k}$ with $1 \leq n_{k+1}-n_{k} \leq 3$ for every $k$ and $\sup _{k} l_{n_{k}}<$ $\infty$. We denote by $S$ the subsequence of every $n_{k}$ verifying $n_{k+1}-n_{k}=3$. We say that $\Omega$ is regular if the following subsequences (with $n_{k} \in S$ ) are regular: $\left\{r_{n_{k}}\right\},\left\{r_{n_{k}+1}\right\},\left\{r_{n_{k}+2}\right\},\left\{l_{n_{k}+1}\right\},\left\{l_{n_{k}+2}\right\},\left\{l_{n_{k}+1}-l_{n_{k}+2}\right\},\left\{l_{n_{k}+2}-\right.$ $\left.l_{n_{k}+1}\right\},\left\{l_{n_{k}+2}-l_{n_{k}+1}+r_{n_{k}+1}\right\},\left\{l_{n_{k}+1}-l_{n_{k}+2}+r_{n_{k}+1}\right\},\left\{l_{n_{k}+1}+l_{n_{k}+2}-\right.$ $\left.r_{n_{k}+1}\right\},\left\{l_{n_{k}+2}-l_{n_{k}+1}+r_{n_{k}}\right\},\left\{l_{n_{k}+1}-l_{n_{k}+2}+r_{n_{k}+2}\right\}$.

Remark 4.2. (1) The maximum and the minimum of several regular sequences is also a regular sequence.

(2) Note that any rational function evaluated in the variables $n^{a_{1}}, \ldots, n^{a_{j}}$, $e^{b_{1} n}, \ldots, e^{b_{j} n},(\log n)^{c_{1}}, \ldots,(\log n)^{c_{j}}$, is regular. In particular, if $\left\{r_{n_{k}}\right\},\left\{r_{n_{k}+1}\right\}$, $\left\{r_{n_{k}+2}\right\},\left\{l_{n_{k}+1}\right\},\left\{l_{n_{k}+2}\right\}$ are rational functions in these variables, then the domain is regular.

Theorem 4.3. Let $\Omega$ be a train such that there exists a subsequence $\left\{n_{k}\right\}_{k}$ with $1 \leq n_{k+1}-n_{k} \leq 3$ for every $k$ and $\sup _{k} l_{n_{k}}<\infty$. Denote by $S$ the subsequence of every $n_{k}$ verifying $n_{k+1}-n_{k}=3$, and assume also that $\Omega$ is regular. Then $\Omega$ is hyperbolic if and only if there exists a constant $C$ such that $\min \left\{l_{n_{k}+1}, r_{n_{k}}, r_{n_{k}+1}\right\} \leq C$ for every $k$ with $n_{k+1}-n_{k}=2$, and we have any of the following conditions:

(a) $\min \left\{\max \left\{r_{n_{k}}, r_{n_{k}+1}\right\}, \max \left\{r_{n_{k}}, r_{n_{k}+2}\right\}, \max \left\{r_{n_{k}+1}, r_{n_{k}+2}\right\}\right\} \leq C$ for every $n_{k} \in S$.

(b) $\min \left\{\max \left\{r_{n_{k}}, l_{n_{k}+2}\right\}, \max \left\{r_{n_{k}+2}, l_{n_{k}+1}\right\}\right\} \leq C$ for every $n_{k} \in S$.

(c) $\max \left\{r_{n_{k}}, l_{n_{k}+2}-l_{n_{k}+1}+r_{n_{k}+1}\right\} \leq C$ for every $n_{k} \in S$, and

$$
\begin{aligned}
\lim _{k \rightarrow \infty, n_{k} \in S} r_{n_{k}+1} & =\lim _{k \rightarrow \infty, n_{k} \in S} l_{n_{k}+2}=\lim _{k \rightarrow \infty, n_{k} \in S}\left(l_{n_{k}+1}-l_{n_{k}+2}\right) \\
& =\lim _{k \rightarrow \infty, n_{k} \in S}\left(l_{n_{k}+1}+l_{n_{k}+2}-r_{n_{k}+1}\right)=\infty .
\end{aligned}
$$

(c') $\max \left\{r_{n_{k}+2}, l_{n_{k}+1}-l_{n_{k}+2}+r_{n_{k}+1}\right\} \leq C$ for every $n_{k} \in S$, and

$$
\begin{aligned}
\lim _{k \rightarrow \infty, n_{k} \in S} r_{n_{k}+1} & =\lim _{k \rightarrow \infty, n_{k} \in S} l_{n_{k}+1}=\lim _{k \rightarrow \infty, n_{k} \in S}\left(l_{n_{k}+2}-l_{n_{k}+1}\right) \\
& =\lim _{k \rightarrow \infty, n_{k} \in S}\left(l_{n_{k}+1}+l_{n_{k}+2}-r_{n_{k}+1}\right)=\infty .
\end{aligned}
$$

(d) $\max \left\{r_{n_{k}+1}, \min \left\{l_{n_{k}+1}, l_{n_{k}+2}\right\}\right\} \leq C$ for every $n_{k} \in S$.

(e) $\max \left\{r_{n_{k}+1}, l_{n_{k}+2}-l_{n_{k}+1}+r_{n_{k}}\right\} \leq C$ for every $n_{k} \in S$, and

$$
\lim _{k \rightarrow \infty, n_{k} \in S} l_{n_{k}+1}=\lim _{k \rightarrow \infty, n_{k} \in S} l_{n_{k}+2}=\infty .
$$

(e') $\max \left\{r_{n_{k}+1}, l_{n_{k}+1}-l_{n_{k}+2}+r_{n_{k}+2}\right\} \leq C$ for every $n_{k} \in S$, and

$$
\lim _{k \rightarrow \infty, n_{k} \in S} l_{n_{k}+1}=\lim _{k \rightarrow \infty, n_{k} \in S} l_{n_{k}+2}=\infty .
$$

(f) $\max \left\{l_{n_{k}+1}, l_{n_{k}+2}\right\} \leq C$ for every $n_{k} \in S$. 
Proof. First of all notice that, by hypothesis, the lengths $l_{n_{k}}$ of the fundamental geodesics $\gamma_{n_{k}}^{*}$ belonging to the subsequence $\left\{n_{k}\right\}_{k}$ satisfy $l_{n_{k}} \leq c$ for some constant $c$. Therefore, if $m \in\left\{n_{k}\right\}_{k}$, then $d_{\Omega}(z, \mathbb{R}) \leq l_{m} \leq c$ for every $z \in \gamma_{m}^{*}$. It means that we just have to care about $d_{\Omega}(z, \mathbb{R})$ for every $z \in\left\{\gamma_{m}^{*}\right\}$ with $m \notin\left\{n_{k}\right\}_{k}$.

By Theorem 3.1 we know that $d_{\Omega}(z, \mathbb{R}) \leq c_{1}$ for every $z \in \gamma_{n_{k}+1}^{*}$ with $n_{k+1}-n_{k}=2$ if and only if $\min \left\{l_{n_{k}+1}, r_{n_{k}}, r_{n_{k}+1}\right\} \leq C$ for every $k$ with $n_{k+1}-n_{k}=2$. Then we just have to deal with $d_{\Omega}(z, \mathbb{R})$ for every $z \in \gamma_{m}^{*}$ with $m \in S$. Therefore, in what follows, we will assume that for all the subsequences involved, the index runs on the values $k$ with $n_{k} \in S$.

By hypothesis we know that every subsequence appearing in the items (a)-(f) either is upper bounded or has infinite limit.

First, let us assume that none of the subsequences $\left\{r_{n_{k}}\right\}_{k},\left\{r_{n_{k}+1}\right\}_{k},\left\{r_{n_{k}+2}\right\}_{k}$ is bounded. In this situation, if $\max \left\{l_{n_{k}+1}, l_{n_{k}+2}\right\}=\infty$, we conclude that $\Omega$ is not hyperbolic by Theorem $3.9(\mathrm{e})$. Otherwise, $\Omega$ is hyperbolic by Theorem $3.10(\mathrm{f})$.

Second, let us assume either that two of the subsequences $\left\{r_{n_{k}}\right\}_{k},\left\{r_{n_{k}+1}\right\}_{k}$, $\left\{r_{n_{k}+2}\right\}_{k}$ are bounded and the other is not bounded, or all three sequences $\left\{r_{n_{k}}\right\}_{k},\left\{r_{n_{k}+1}\right\}_{k},\left\{r_{n_{k}+2}\right\}_{k}$ are bounded. Then, by Theorem 3.10(a), $\Omega$ is hyperbolic.

Hence, from now on, we will assume that only one of the previously mentioned subsequences is bounded. If this is the situation, we have to distinguish two different cases: the first one occurs when either $\left\{r_{n_{k}}\right\}_{k}$ or $\left\{r_{n_{k}+2}\right\}_{k}$ is bounded, and the second one, when $\left\{r_{n_{k}+1}\right\}_{k}$ is the bounded subsequence. Let us deal with the first case, but we will just analyze the first possibility, i.e., when the bounded sequence is $\left\{r_{n_{k}}\right\}_{k}$, since the remaining case is symmetric. Notice that, if $l_{n_{k}+2} \leq C$ for every $k$, then $\Omega$ is hyperbolic by Theorem 3.10 (b). If this is not the case, i.e., if $\lim _{k \rightarrow \infty} l_{n_{k}+2}=\infty$, then we will again distinguish two possibilities:

(1) If $l_{n_{k}+1}-l_{n_{k}+2} \leq C$ for every $k$, then by Theorem $3.9(\mathrm{a}), \Omega$ is not hyperbolic.

(2) Otherwise, i.e., if $\lim _{k \rightarrow \infty}\left(l_{n_{k}+1}-l_{n_{k}+2}\right)=\infty$, we have to consider two options:

(a) If $l_{n_{k}+1}+l_{n_{k}+2}-r_{n_{k}+1} \leq C$ for every $k$, then, by Theorem 3.9 (b), $\Omega$ is not hyperbolic.

(b) If the expression mentioned above tends to infinity with $k$, then we have two alternatives:

(i) If $l_{n_{k}+2}-l_{n_{k}+1}+r_{n_{k}+1} \leq C$ for every $k$, then $\Omega$ is hyperbolic by Theorem $3.10(\mathrm{c})$.

(ii) If the expression mentioned above tends to infinity with $k$, then $\Omega$ is not hyperbolic by Theorem 3.9(c).

This covers all the possibilities when the subsequence $\left\{r_{n_{k}}\right\}_{k}$ (or $\left\{r_{n_{k}+2}\right\}_{k}$ ) is bounded. Let us study now what happens when the only bounded subsequence 
is $\left\{r_{n_{k}+1}\right\}_{k}$. If this is the situation, and besides one of the two subsequences $\left\{l_{n_{k}+1}\right\}_{k}$ or $\left\{l_{n_{k}+2}\right\}_{k}$ is bounded, then $\Omega$ is hyperbolic by Theorem $3.10(\mathrm{~d})$. Otherwise, i.e., when both subsequences tend to infinity with $k$, we distinguish two cases:

(1) If $l_{n_{k}+2}-l_{n_{k}+1}+r_{n_{k}} \leq C$ for every $k$, then $\Omega$ is hyperbolic by Theorem $3.10(\mathrm{e})$.

(2) If $\lim _{k \rightarrow \infty}\left(l_{n_{k}+2}-l_{n_{k}+1}+r_{n_{k}}\right)=\infty$, there are, again, two possibilities: (a) If $l_{n_{k}+2}-l_{n_{k}+1} \leq C$ for every $k, \Omega$ is not hyperbolic by Theorem $3.9(\mathrm{~d})$.

(b) If the expression mentioned above tends to infinity with $k$, it means that $\left\{l_{n_{k}+1}-l_{n_{k}+2}\right\}_{k}$ is upper bounded, and then there are two options:

(i) If $\lim _{k \rightarrow \infty}\left(l_{n_{k}+1}-l_{n_{k}+2}+r_{n_{k}+2}\right)=\infty$, then $\Omega$ is not hyperbolic by Theorem 3.9(d').

(ii) Otherwise, $l_{n_{k}+1}-l_{n_{k}+2}+r_{n_{k}+2} \leq C$ for every $k$, and $\Omega$ is hyperbolic by Theorem 3.10(e').

This finishes the proof.

\section{References}

[1] H. Aikawa, Positive harmonic functions of finite order in a Denjoy type domain, Proc. Amer. Math. Soc. 131 (2003), no. 12, 3873-3881.

[2] V. Alvarez, D. Pestana, and J. M. Rodríguez, Isoperimetric inequalities in Riemann surfaces of infinite type, Rev. Mat. Iberoamericana 15 (1999), no. 2, 353-427.

[3] V. Alvarez, A. Portilla, J. M. Rodríguez, and E. Tourís, Gromov hyperbolicity of Denjoy domains, Geom. Dedicata 121 (2006), 221-245.

[4] V. Alvarez, J. M. Rodríguez, and D. A. Yakubovich, Estimates for nonlinear harmonic "measures" on trees, Michigan Math. J. 49 (2001), no. 1, 47-64.

[5] J. W. Anderson, Hyperbolic Geometry, Springer, London, 1999.

[6] Z. M. Balogh and S. M. Buckley, Geometric characterizations of Gromov hyperbolicity, Invent. Math. 153 (2003), no. 2, 261-301.

[7] A. Basmajian, Constructing pairs of pants, Ann. Acad. Sci. Fenn. Ser. A I Math. 15 (1990), no. 1, 65-74.

[8] , Hyperbolic structures for surfaces of infinite type, Trans. Amer. Math. Soc. 336 (1993), no. 1, 421-444.

[9] A. F. Beardon, The Geometry of Discrete Groups, Springer-Verlag, New York, 1983.

[10] L. Bers, An inequality for Riemann surfaces, Differential geometry and complex analysis, 87-93, Springer, Berlin, 1985.

[11] M. Bonk, J. Heinonen, and P. Koskela, Uniformizing Gromov hyperbolic spaces, Asterisque No. 270 (2001), viii+99 pp.

[12] A. Cantón, J. L. Fernández, D. Pestana, J. M. Rodríguez, On harmonic functions on trees, Potential Anal. 15 (2001), no. 3, 199-244.

[13] M. Coornaert, T. Delzant, and A. Papadopoulos, Notes sur les groups hyperboliques de Gromov, I.R.M.A., Strasbourg, 1989.

[14] J. L. Fernández and J. M. Rodríguez, Area growth and Green's function of Riemann surfaces, Ark. Mat. 30 (1992), no. 1, 83-92.

[15] J. Garnett and P. Jones, The corona theorem for Denjoy domains, Acta Math. 155 (1985), no. 1-2, 27-40. 
[16] E. Ghys and P. de la Harpe, Panorama, Sur les groupes hyperboliques d'après Mikhael Gromov (Bern, 1988), 1-25, Progr. Math., 83, Birkhäuser Boston, Boston, MA, 1990.

[17] M. J. González, An estimate on the distortion of the logarithmic capacity, Proc. Amer. Math. Soc. 126 (1998), no. 5, 1429-1431.

[18] M. Gromov, Hyperbolic groups, Essays in group theory, 75-263, Math. Sci. Res. Inst. Publ., 8, Springer, New York, 1987.

[19] _ Metric Structures for Riemannian and Non-Riemannian Spaces, With appendices by M. Katz, P. Pansu and S. Semmes. Translated from the French by Sean Michael Bates. Progress in Mathematics, 152. Birkhauser Boston, Inc., Boston, MA, 1999.

[20] A. Haas, Dirichlet points, Garnett points, and infinite ends of hyperbolic surfaces. I, Ann. Acad. Sci. Fenn. Math. 21 (1996), no. 1, 3-29.

[21] P. A. Hästö, Gromov hyperbolicity of the $j_{G}$ and $\tilde{j}_{G}$ metrics, Proc. Amer. Math. Soc. 134 (2006), no. 4, 1137-1142.

[22] P. A. Hästö, H. Lindén, A. Portilla, J. M. Rodríguez, E. Tourís, Gromov hyperbolicity of Denjoy domains with hyperbolic and quasihyperbolic metrics, To appear in J. Math. Soc. Japan.

[23] P. A. Hästö, A. Portilla, J. M. Rodríguez, and E. Tourís, Gromov hyperbolic equivalence of the hyperbolic and quasihyperbolic metrics in Denjoy domains, Bull. Lond. Math. Soc. 42 (2010), 282-294.

[24] Comparative Gromov hyperbolicity results for the hyperbolic and quasihyperbolic metrics, Complex Var. Elliptic Equ. 55 (2010), no. 1-3, 127-135.

[25] Uniformly separated sets and Gromov hyperbolicity of domains with the quasihyperbolicity metric, To appear in Medit. J. Math.

[26] _ Gromov hyperbolicity of Denjoy domains through fundamental domains, Submitted.

[27] I. Holopainen and P. M. Soardi, p-harmonic functions on graphs and manifolds, Manuscripta Math. 94 (1997), no. 1, 95-110.

[28] M. Kanai, Rough isometries, and combinatorial approximations of geometries of noncompact Riemannian manifolds, J. Math. Soc. Japan 37 (1985), no. 3, 391-413.

[29] , Rough isometries and the parabolicity of Riemannian manifolds, J. Math. Soc. Japan 38 (1986), no. 2, 227-238.

[30] - Analytic inequalities, and rough isometries between noncompact Riemannian manifolds, Curvature and topology of Riemannian manifolds (Katata, 1985), 122-137, Lecture Notes in Math., 1201, Springer, Berlin, 1986.

[31] A. Karlsson and G. A. Noskov, The Hilbert metric and Gromov hyperbolicity, Enseign. Math. (2) 48 (2002), no. 1-2, 73-89.

[32] H. Lindén, Gromov hyperbolicity of certain conformal invariant metrics, Ann. Acad. Sci. Fenn. Math. 32 (2007), no. 1, 279-288.

[33] F. Paulin, On the critical exponent of a discrete group of hyperbolic isometries, Differential Geom. Appl. 7 (1997), no. 3, 231-236.

[34] A. Portilla, J. M. Rodríguez, and E. Tourís, Gromov hyperbolicity through decomposition of metrics spaces. II, J. Geom. Anal. 14 (2004), no. 1, 123-149.

[35] _ The topology of balls and Gromov hyperbolicity of Riemann surfaces, Differential Geom. Appl. 21 (2004), no. 3, 317-335

[36] The role of funnels and punctures in the Gromov hyperbolicity of Riemann surfaces, Proc. Edinb. Math. Soc. (2) 49 (2006), no. 2, 399-425.

[37] Stability of Gromov hyperbolicity, J. Adv. Math. Stud. 2 (2009), no. 2, 77-96.

[38] _ A real variable characterization of Gromov hyperbolicity of flute surfaces, To appear in Osaka J. Math.

[39] A. Portilla and E. Tourís, A characterization of Gromov hyperbolicity of surfaces with variable negative curvature, Publ. Mat. 53 (2009), no. 1, 83-110. 
[40] J. M. Rodríguez, Isoperimetric inequalities and Dirichlet functions of Riemann surfaces, Publ. Mat. 38 (1994), no. 1, 243-253.

[41] _ Two remarks on Riemann surfaces, Publ. Mat. 38 (1994), no. 2, 463-477.

[42] J. M. Rodríguez and J. M. Sigarreta, Location of geodesics and isoperimetric inequalities in Denjoy domains, Submitted.

[43] J. M. Rodríguez and E. Tourís, Gromov hyperbolicity through decomposition of metric spaces, Acta Math. Hungar. 103 (2004), no. 1-2, 107-138.

[44] A new characterization of Gromov hyperbolicity for negatively curved surfaces, Publ. Mat. 50 (2006), no. 2, 249-278.

[45] _ Gromov hyperbolicity of Riemann surfaces, Acta Math. Sin. (Engl. Ser.) 23 (2007), no. 2, 209-228

[46] P. M. Soardi, Rough isometries and Dirichlet finite harmonic functions on graphs, Proc. Amer. Math. Soc. 119 (1993), no. 4, 1239-1248.

[47] E. Tourís, Graphs and Gromov hyperbolicity of non-constant negatively curved surfaces, To appear in J. Math. Anal. Appl..

Ana Portilla

St. Louis University (Madrid Campus)

AVEnida DEL VALle 34

28003 MADRID, SPAIN

E-mail address: aportil2@slu.edu

José M. RodRÍGuez

Departamento de Matemáticas

Escuela Politécnica Superior

Universidad CARlos III DE MAdRID

Avenida DE LA Universidad, 30

28911 Leganés (MAdrid), SPAin

E-mail address: jomaro@math.uc3m.es

Eva TOURÍs

Departamento de Matemáticas

Escuela Politécnica Superior

Universidad Carlos III de Madrid

AVEnida DE LA Universidad, 30

28911 LeganÉs (MAdrid), Spain

E-mail address: etouris@math.uc3m.es 